\title{
Commentary: The Norwood operation from 1982 to 2019: Continued surgical triumph or stagnation?
}

\author{
Emile A. Bacha, MD
}

\footnotetext{
From the Division of Cardiac, Thoracic and Vascular Surgery, New York-Presbyterian/Morgan Stanley Children's Hospital, Columbia University Medical Center, Pediatric and Congenital Cardiac Surgery, New York, NY. Disclosures: Author has nothing to disclose with regard to commercial support.

Received for publication Jan 21, 2019; accepted for publication Jan 21, 2019; available ahead of print March 7 , 2019.

Address for reprints: Emile A. Bacha, MD, Department of Cardiac Surgery, Columbia University, Morgan Stanley Children's Hospital, New York, NY 10032 (E-mail: eb2709@cumc.columbia.edu).

J Thorac Cardiovasc Surg 2019;158:230-94

$0022-5223 / \$ 36.00$

Copyright (c) 2019 by The American Association for Thoracic Surgery

https://doi.org/10.1016/j.jtcvs.2019.01.074
}

The seminal New England Journal of Medicine article by Norwood and colleagues ${ }^{1}$ in 1983 , detailing the first successful eponymous stage I palliation procedure performed at Children's Hospital Boston, should be required reading for anybody dealing with patients who have congenital heart disease. It can serve as a blueprint to anyone interested in clinical innovation. It does not boast of a single great "eureka" innovative moment of when the stage I procedure for hypoplastic left heart syndrome (HLHS) was born. Instead, it describes an iterative scientific process focused on one lesion and one problem, namely, that HLHS did not have (and still does not have) a known cure. In addition to describing the technical details of the procedure, Norwood and colleagues ${ }^{1}$ also detail the circumstances, the programmatic changes, and the culture that allowed this to occur, which includes the introduction of the neonatal arterial switch operation in 1980, also in Boston. The foundational physiology of the stage I procedure (unobstructed systemic outflow, unrestricted atrial septum and pulmonary venous return, restricted pulmonary blood flow) is described in definitive terms. It is striking, when one rereads the early literature of that time, how much and how little have actually changed at the same time. The stage I procedure has been tweaked and modified many times, yet its basic tenets remain the same. Dr Norwood subsequently moved to the Children's Hospital of Philadelphia in 1984, and that is where Mascio and colleagues ${ }^{2}$ start their retrospective review. With a total of 1663 Norwood procedures performed for HLHS and other single-ventricle malformations over a 30-year period, they have collected the largest series to date and have confirmed what numerous other studies have shown, namely, that results improved significantly over time (worse era 1984-1988; best era 2004-2008). They also showed that in univariate modeling, anomalous pulmonary venous drainage, moderate or severe atrioventricular regurgitation, lower weight, prematurity, genetic anomaly, and race other than white are risk factors for mortality. In multivariate analysis, only anomalous

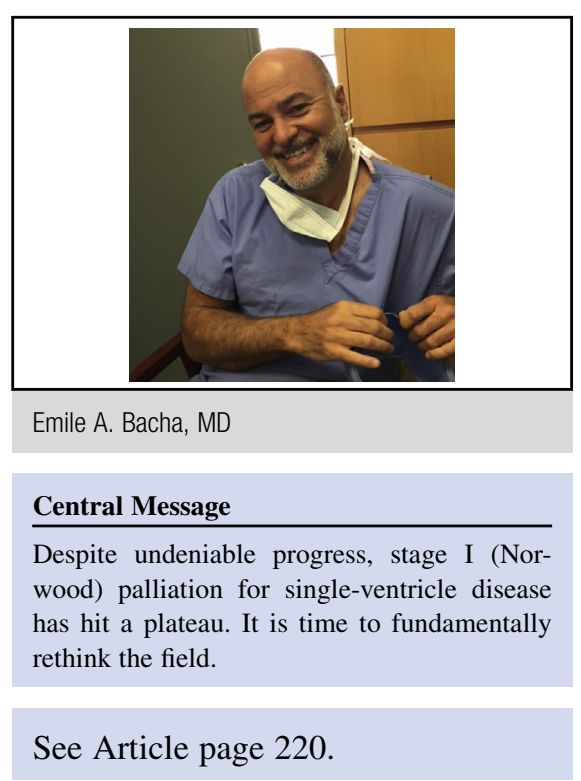

pulmonary venous drainage, prematurity, genetic anomaly, and race remained significant. Ascending aortic size, age at surgery, and HLHS subtype were not associated with risk of mortality. A novel finding was that the impact of risk factors was found to be cumulative, and mortality increased as the number of risk factors increased. The decline in mortality over the years was due to increasing experience with the operation, introduction of prenatal diagnosis, and improvements in perioperative care. The plateauing in survival of the recent 10 years was explained by the increasing proportion of high-risk patients, which is itself a consequence of improved maternal-fetal and neonatal care, resulting in more premature and complex neonates surviving to the operation. Finally, they mention that the identified risk factors are unfortunately not modifiable.

Amassing data on patients over 30 years is a formidable undertaking, and the authors should be congratulated on keeping detailed records, a cornerstone of quality management in congenital heart surgery. When reading the article, one cannot escape a feeling of a missed opportunity however. Thirty years is a long time, and surely, after 30 years of institutional experience, we should be able to expect specific "lessons learned" from an institution at the vanguard of HLHS management. These might include simply their own ideal management under various clinical scenarios. More specifically, this information might also include the outcomes of Norwood cases with a Blalock-Taussig shunt 
versus right ventricle to pulmonary artery conduits (the last such publication by Children's Hospital of Philadelphia is from $2005^{3}$ ), the shunt size, the intraoperative strategies (deep hypothermic circulatory arrest, temperature, $\mathrm{pH}$ stat vs alpha stat), the role of individual surgeons, and some information on interstage mortality or (ideally) 1 -year survival, to name a few. Indeed, as is increasingly clear, hospital mortality alone after the stage I procedure is becoming increasingly less meaningful.

The role of the individual surgeon on outcomes particularly can be explored well here, because this is going to be as close as we ever get to a scientific experiment of sorts, that is, having different surgeons operate on a sufficient number of patients in a nearly uniform perioperative environment to actually potentially see significant differences. These differences could inform us as to best approaches to the Norwood operation. Multicenter studies, with their varied perioperative approaches, will never be able to match that scenario. In addition, the reality is that in the future, patients requiring a Norwood operation will continue to be increasingly small and complex. If we as a profession want to be ready and push the field forward, these detailed analyses will have to be done. Thirty-six years after the first successful Norwood operation was performed, we are still dealing with the same plateaued early survival numbers, a $20 \%$ rate of serious neurologic events, a 5-year survival in the range of $65 \%$, and adult survivors with liver fibrosis and requiring high-risk transplants. Dr Norwood showed the way of true innovation back then; it is time for others to pick up that mantle.

\section{References}

1. Norwood WI, Lang P, Hansen DD. Physiologic repair of aortic atresia-hypoplastic left heart syndrome. Physiologic repair of aortic atresia-hypoplastic left heart syndrome. N Engl J Med. 1983;308:23-6.

2. Mascio CE, Irons ML, Ittenbach RF, Gaynor JW, Fuller SM, Kaplinski M, et al Thirty years and 1663 consecutive Norwood procedures: has survival plateaued? J Thorac Cardiovasc Surg. 2019;158:220-9.

3. Tabbutt S, Dominguez TE, Ravishankar C, Marino BS, Gruber PJ, Wernovsky G, et al. Outcomes after the stage I reconstruction comparing the right ventricular to pulmonary artery conduit with the modified Blalock Taussig shunt. Ann Thorac Surg. 2005;80:1582-90.

4. Newburger JW, Sleeper LA, Gaynor JW, Hollenbeck-Pringle D, Frommelt PC, Li JS, et al. Transplant-free survival and interventions at 6 years in the SVR trial. Circulation. 2018;137:2246-53. 